\title{
RECENT PWAS-SHM DEVELOPMENTS IN THE LABORATORY FOR ACTIVE MATERIALS AND SMART STRUCTURES
}

\author{
Victor Giurgiutiu \\ University of South Carolina \\ Columbia, SC 29208 \\ victorg@sc.edu
}

\section{ABSTRACT}

This paper will present recent results obtained in the Laboratory for Active Materials and Smart Structures in the development of piezoelectric wafer active sensors (PWAS) technologies for structural health monitoring (SHM) applications. One development consists in a carefully conducted study of the convergence of commercially available finite element method (FEM) codes used in the simulation of guided waves traveling in thin-wall structures. Furthermore, the interaction of these waves with various types of damage was studied with the FEM method and compared with experimental results. Another development consists in an analytical model for the prediction of the interaction between PWAS-generated guided waves and structural damage. This topic has been developed and coded in a MATLAB GUI under the name of Wave Form Revealer (WFR). The WFR software allows for the linear and nonlinear analysis of multimodal guided waves in interaction with various damage types characterized by damage interaction parameters. The study of energy transduction between the PWAS and thin-wall structure subjected to guided wave SHM was undertaken in the context of multiple guided wave modes and very interesting energy tuning principles for optimal power consumption have been developed. The paper ends with conclusions and suggestions for further work.

\section{INTRODUCTION}

Structural health monitoring (SHM) sets forth to determine the health of a structure by monitoring over time a set of structural sensors; this yields an assessment of the remaining useful life and the need for structural actions. Built-in SHM system capable of detecting and quantifying damage would increase the operational safety and reliability, would conceivably reduce the number of unscheduled repairs, and would bring down maintenance cost. SHM sensors should be able to actively interrogate the structure and find out its state of health, its remaining life, and the effective margin of safety.
Two SHM sensing principles can be considered: (a) passive SHM sensing, in which the damage of the structure is inferred from the changes in load and strain distributions measured by the sensors; and (b) active SHM sensing, in which the damage is sensed by active interrogation of the structure with elastic waves. The Laboratory for Active Materials and Smart Structures (LAMSS) at the University of South Carolina has accumulated results and experience in developing active SHM sensors and systems. This paper covers briefly some of these results as presented next.

\section{PIEZOELECTRIC WAFER ACTIVE SENSORS}

Piezoelectric wafer active sensors (PWAS) are two-way transducers [1] that couple the electrical and mechanical effects (mechanical strain, $S_{i j}$, mechanical stress, $T_{k l}$, electrical field, $E_{k}$, and electrical displacement, $D_{j}$ ) through the tensorial piezoelectric constitutive equations

$$
\begin{aligned}
& S_{i j}=s_{i j k l}^{E} T_{k l}+d_{k i j} E_{k} \\
& D_{j}=d_{j k l} T_{k l}+\varepsilon_{j k}^{T} E_{k}
\end{aligned}
$$

where, $s_{i j k l}^{E}$ is the mechanical compliance of the material measured at zero electric field $(E=0), \varepsilon_{j k}^{T}$ is the dielectric permittivity measured at zero mechanical stress $(T=0)$, and $d_{k i j}$ represents the piezoelectric coupling effect. PWAS utilize the $d_{31}$ coupling between in-plane strains, $S_{1}, S_{2}$, and transverse electric field, $E_{3}$. PWAS are transducers are different from conventional ultrasonic transducers in several way as described in ref. [1]:

By using Lamb waves in "thin-wall" structures such as piping and pressure vessels, one can detect structural anomaly, i.e., cracks, corrosions, delaminations, and other damage.

PWAS transducers can serve several purposes [1]: (a) highbandwidth strain sensors; (b) high-bandwidth wave exciters and 
receivers; (c) resonators; (d) embedded modal sensors with the electromechanical (E/M) impedance method. By application types, PWAS transducers can be used for (i) active sensing of far-field damage using pulse-echo, pitch-catch, and phasedarray methods, (ii) active sensing of near-field damage using high-frequency E/M impedance method and thickness-gage mode, and (iii) passive sensing of damage-generating events through detection of low-velocity impacts and acoustic emission at the tip of advancing cracks (Figure 1). PWAS phased arrays permit broadside and offside cracks to be independently identified with scanning beams emitting from a central location. The main advantage of PWAS over conventional ultrasonic probes is in their small size, lightweight, low profile, and small cost. In spite of their small size, PWAS are able to replicate many of the functions performed by conventional ultrasonic probes.

Several questions arise when considering the use of PWAS transducers in SHM applications:

(a) Given that finite element method (FEM) is widely used for solving engineering problems, how good and efficient is the FEM simulation for guided wave propagation in the ultrasonic range? What convergence guidelines should we follow in the FEM mesh in order to capture correctly the guided waves behavior at high frequencies (i.e., small wavelengths) and its interaction with small damage?

(b) How much ultrasonic wave power can one inject in the structure with a PWAS transducer of a given size operating at a given voltage and frequency? Are there optimal ways of designing the PWAS transducer such that it couples best with the structure for transmitting ultrasonic guided waves of a certain type? (can we achieve and effect like 'impedance matching' and 'wavelength tuning' in radio waves engineering?)

(c) Can one optimize the placing of the PWAS transducers such as to minimize their number while maximizing their capability to detect damage? Do we have the capability of simulating the in the ultrasonic guided waves signal changes that are due to a certain type of damage in a given structure? Are these simulations efficient such that we can run parameter studies to design an optimum SHM system?

(d) Is there a way to combine the efficiency of analytical methods with the ease of use of FEM approaches such that to enable the running of large parameter studies and in optimization loops?

(e) Given that composite materials are gaining more and more acceptance in engineering, how should the active SHM approach be modified to take into account the complications introduced by the composite anisotropy and multi-orientation layup in the modeling of ultrasonic guided wave propagation?

The present paper will address some of these questions and present recent results obtained in LAMSS at USC.

\section{CONVERGENCE GUIDELINES FOR FEM USE IN GUIDED WAVES PREDICTIVE SIMULATION}

We have systematically studied the convergence of the FEM approach when predicting the propagation of ultrasonic guide waves in a thin-wall structure [2],[3]. The package used in the present study, ABAQUS/Explicit, uses an explicit integration based on a central difference method. The stability of the numerical solution dependents upon the time and the space resolutions of the analysis. ABAQUS/Explicit recommends a numerical stability limit for the integration time step is

$$
\Delta t \leq \frac{1}{20 f_{\max }}
$$

The maximum frequency of the dynamic problem, $f_{\max }$ limits both the integration time step and the element size. The size of the finite element, $L_{e}$ is typically derived from the smallest wavelength to be analyzed, $\lambda_{\min }$. For a good spatial resolution 20 nodes per wavelength are recommended. This condition can be written as

$$
L_{e} \leq \frac{\lambda_{\min }}{20}
$$

In active SHM, both the symmetric and antisymmetric guided wave modes are used. The fundamental antisymmetric mode A0 is highly dispersive and frequencies below $100 \mathrm{kHz}-\mathrm{mm}$ since its wave speed tends to zero at very low frequencies. Since at a given frequency, the A0 wavelength is much shorter than that of the corresponding symmetric mode S0 and since A0 mode is much more dispersive, the FEM simulation of the A0 mode requires fine spatial discretization with substantial computational cost. In contrast, the S0 mode has a bigger wavelength and is much less dispersive. We express the mesh density as $N=\lambda / L_{e}$ (nodes per wavelength). Figure $2 \mathrm{a}$ highlights the strong influence of the mesh density on the group velocity error for the A0 mode during the simulation of straight-crested guided wave propagation using 2-D FEM meshing [2]. The curve shows how the error varies from a value of about $9 \%$ for $\mathrm{N}=15$ to a value of $0.2 \%$ for $\mathrm{N}=254$ for this mode. For the fundamental symmetric mode S0, the error varies from about $2 \%$ for $\mathrm{N}=20$ to $0.15 \%$ for $\mathrm{N}=120$.

For the simulation of circular-crested guided wave propagation, we used 3-D FEM meshing and the ABAQUS/explicit solver. We discretized the plate with C3D8R brick elements of size $\lambda_{e}=0.5 \mathrm{~mm}$. We applied selfequilibrating forces to simulate the excitation from square and round PWAS transducers as shown in Figure 2b,c. A $150-\mathrm{kHz}$ 3 -count smoothed tone burst was used as excitation. The simulated wave signals, which contain both S0 and A0 wave packets, were compared with 1-D analytical simulations [4] and 2-D experiments performed on a large 3.2-mm thick aluminum plate with various paths between the transmitter and receiver PWAS transducers [3]. 
The analytical, finite element and experimental results for the path P1-P2 having 200-mm distance between the transmitter and receiver PWAS transducers are shown in Figure 3. A good matching is observed between the FEM and the experimental results for both $\mathrm{S} 0$ and $\mathrm{A} 0$ packets. However, the matching of the analytical prediction is not as good, especially for the S0 packet which displays phase shift and larger amplitude (Figure 3a). However, the introduction of the shear lag correction for the attachment between PWAS and structure [5] has corrected this issue as shown in Figure 3b. Another difference is the slight time shift observed at the end of the A0 packet between the three different methods. This may be due to the approximation of the excitation in the analytical and the FEM model compared with the actual experiments.

After these calibration studies, the same FEM meshing was slightly modified to simulate structural damage (small notches) and then used to predict the guided wave signals transmitted through and reflected from this damage [3].

\section{WAVE FORM REVEALER - AN ANALYTICAL PREDICTIVE TOOL FOR THE SIMULATION OF MULTIMODAL GUIDED WAVES PROPAGATION AND INTERACTION WITH DAMAGE}

An analytical approach based on the 1D (straight crested) guided wave propagation analysis was used to predict the ultrasonic guided wave propagation in thin-wall structures. This analytical approach was coded into MATLAB and the WaveFormRevealer (WFR) graphical user interface (GUI) was developed [4]. In addition to guided wave propagation, the WFR code can also predict the guided wave interaction with damage. This was attained by inserting the damage effect as transmission, reflection, mode conversion, and higher harmonics damage interaction coefficients that act at the damage site. The WFR code does not derive these damage interaction coefficients, but imports them from external sources (either available literature data or FEM analysis performed in a separate computational module).

The WFR can generate fast predictions of waveforms resulting from Lamb waves' interaction with damage for arbitrary positioning of PWAS transmitters and receivers with respect to damage and with respect to each other. The users may choose their own excitation signal, PWAS size, structural parameters, and damage description. The current version of the WFR code is limited to 1D (straight crested) guided wave propagation; extension of this approach to $2 \mathrm{D}$ (circular crested) guided wave propagation is under development.

Figure 4 shows how an electrical tone burst applied to a transmitter PWAS transducer (T-PWAS) propagates through a structural waveguide to the receiver PWAS transducer (RPWAS) in pitch-catch mode. When the incident wave encounters the damage, part of it will be transmitted and part of it will be reflected. If the damage is symmetric across the thickness, then an incident S0 wave will result into a transmitted S0 wave and a reflected S0 wave. Thus, the damage acts as a new wave source. But if the damage is not symmetric across the thickness, then mode conversion will also take place because guided waves are inherently multimodal. If mode conversation is present, then an incident S0 wave will result in transmitted and reflected $\mathrm{A} 0$ waves as well as transmitted and reflected S0 waves. If the incident wave is A0, a similar mode conversion will take place. If the incident wave contains both S0 and A0 waves, then the multimodal transmission and reflection process illustrated in Figure 6 will take place.

In addition to linear damage interaction processes, the WFR code also considers nonlinear damage interaction situations that results in higher harmonics being generated, as illustrated in Figure 5. The nonlinear effect creates higher harmonics that can be detected through spectral analysis of the signal, which is also done in the WFR code.

\section{TWO-WAY POWER AND ENERGY TRANSDUCTION BETWEEN PWAS AND STRUCTURE}

A systematic investigation of power and energy transduction between PWAS and structure during the structural health monitoring process has been undertaken. The investigation was performed with analytical tools because they are more efficient than the finite element method (FEM) in performing parametric studies and thus highlighting trends and directions for transduction optimization. In these analytical studies, the model was restricted to conventional axial and flexural waves; although this model is not rigorously correct for high-frequency ultrasonic waves, it was adopted for its computational efficiency. In future investigations, we intend to replace this restrictive axial-flexural assumption with the exact unrestricted guided-waves formulation.

The investigation was started with the study of a 1-D analytical model [6] corresponding to straight crested waves. These 1-D results were discussed in our 2010 PVP conference presentation [7]; they will not be repeated here. More recently, we have developed a 2-D model that is more appropriate for the analysis of circular-crested waves [8], which are of great importance for practical implementation because most SHM applications use PWAS located in a 2-D geometry with circular crested waves emanating from a PWAS transmitter and traveling through the structure to the PWAS receiver. In this present paper, we will summarize this work and highlight the main findings.

Our aim was to capture the power and energy flow from the electrical source energizing the transmitter PWAS through various stages of transduction up to the signal captured by an instrument connected to the receiver PWAS. The following energy conversion stages were considered (Figure 7): (a) piezoelectric transduction between source and transmitter PWAS; (b) mechanical transmission of shear stresses from the PWAS to the structure; (c) excitation of ultrasonic waves traveling through the structure from the transmitter to the receiver; (d) capturing of ultrasonic waves arriving at the receiver location; (e) mechanical conversion of structural waves into shear stresses acting from the structure onto the receiver PWAS; (f) piezoelectric conversion at the receiver PWAS and 
measurement by the electrical instrument. The model was used to simulate a pitch-catch SHM process; it can be also used to simulate energy harvesting from structural waves.

The analytical model was developed under the following assumptions: (a) 2-D propagation of axial and flexural waves; (b) ideal bonding (line-force model) between PWAS and structure; (c) ideal voltage excitation source at the transmitter PWAS; (d) external impedance load at the receiver PWAS to represent the measuring instrument or the energy harvester, as appropriate.

Figure 8 presents this 2-D model showing a PWAS transmitter and a PWAS receiver bonded to the surface of an isotropic plate. We assume the circular PWAS transmitter is of radius $a$ located at the origin of a polar coordinate system and it produces axisymmetric circular waves under electrical excitation. A circular PWAS receiver with the center at $\left(r_{s}, 0\right)$

and radius $c$ converts the waves back to electrical signal.

The power and energy transduction flow chart for a complete pitch-catch setup (Figure 7) explains how the twoway electro-acoustic power and energy transduction of the PWAS transmitter and receiver are taking place. In pitch-catch mode, the power flow converts from electrical source into piezoelectric power at the transmitter, the piezoelectric transduction converts the electrical power into the mechanical interface power at the transmitter PWAS and then into acoustic wave power travelling in the structure. The wave power arrives at the receiver PWAS and is captured at the mechanical interface between the receiver PWAS at the structure, the mechanical power captured is converted back into electrical power in the receiver PWAS and captured at the receivers electric instrument. The time-averaged electrical power, mechanical power at the transmitter and wave power can be calculated from the frequency response function. The timeaveraged mechanical power and electrical power at the receiver PWAS can be calculated as well. The main difference between 1-D and 2-D analyses is that the 2-D analysis considered in this paper includes geometric spreading of the power density as the circular crested wave front expand according to the $1 / r$ law, whereas the 1-D analysis considers that the power density is constant in straight crested wave front.

A parametric study of transmitter PWAS size effect of power attached to an aluminum plate was undertaken. Numerical simulation was performed with the parameters given in Table 1. Constant $10-\mathrm{V}$ excitation voltage from an ideal electrical source was assumed at the transmitter PWAS. The circular PWAS radius was varied from 2 to $15 \mathrm{~mm}$, whereas the frequency was spanned from 1 to $1,000 \mathrm{kHz}$.

Figure 9 presents the results of a parameter study of various PWAS sizes and frequencies. The resulting parameter plots are presented as 3D mesh plots. Figure 9a shows a 3D mesh plot of the power rating vs. frequency and transmitter size: for a certain circular transmitter radius, the power rating increases when the frequency increases; for a given frequency, the power rating increases when the transmitter size increases. These results demonstrate that the driving of a $15-\mathrm{mm}$ radius circular PWAS at $1,000 \mathrm{kHz}$ with a $10 \mathrm{~V}$ constant voltage input, one requires $10 \mathrm{~W}$ of power to be provided by the power source. Figure $9 \mathrm{~b}$ shows the wave power that a circular PWAS generates into the structure; tuning effect of transmitter radius and excitation frequency are apparent; a larger PWAS does not necessarily produce more wave power at a given frequency! The maximum wave power output in this simulation is $\sim 20 \mathrm{~mW}$. The wave power is the same as the electrical active power. This study provides guidelines for the design of transmitter size and excitation frequency in order to obtain maximum wave power into the monitored structure during the SHM process.

The power contained in the axial and flexural waves are presented separately in Figure 9c and Figure 9d. In some PWAS SHM applications, a single mode is often desired to reduce signal complexity and simplify signal interpretation and damage detection. Figure 9c shows the frequency-size combinations at which the axial waves are maximized, whereas Figure 9d indicates the combinations that would maximize the flexural waves. These figures give useful guidelines for choosing PWAS size and frequency values that are optimum for selecting a certain excitation wave mode.

The model was further used to predict the frequency response functions for voltage, current, complex power, active power, etc. To facilitate understanding, the simpler case of a PWAS transmitter was considered first. At the input side, it was found that the reactive electric power is dominant and hence defines the size of the energizing power supply/amplifier.

At the PWAS structure interface, it was found that only the active electrical power gets converted into mechanical power, which is transmitted across the PWAS-structure interface and energizes the axial and flexural waves propagating into the structure. A parametric study was conducted w.r.t. the transmitter PWAS size: it was found that proper size and excitation frequency selection facilitates ultrasonic waves excitation through tuning effects. Figure 9b,c,d, shows that a larger PWAS does not necessarily ensure more power transmission -- careful frequency-size tuning is necessary! Similar tuning effects were also found at the receiver PWAS where a parametric study of receiver size, receiver impedance and external electrical load provides useful design guidelines for PWAS-based sensing and/or energy harvesting.

Finally, the power flow for a pitch-catch situation was considered: in this case, the power flows as follows: (a) from the electrical source into the transmitter PWAS; (b) through piezoelectric transduction, into the mechanical power; (c) into ultrasonic wave power through the interface between the transmitter PWAS and the structure; (d) the ultrasonic wave power travels through the structure to the receiver PWAS; (e) the wave power arriving at the receiver PWAS is captured at the mechanical interface between the receiver PWAS and the structure; (f) the captured mechanical power is converted into electrical power at the receiver PWAS through the piezoelectric effect; (g) the electric power is measured by electrical instrument connected at the receiver PWAS. Numerical simulation and graphical charts showed that power and energy 
flow have peaks and valleys that can be utilized for design optimization [6].

\section{SUMMARY AND CONCLUSIONS}

The piezoelectric wafer active sensors (PWAS) presented in this paper are a promising technology for SHM systems Although PWAS transducers have been successful in laboratory tests, several fundamental scientific challenges remain in the implementation of PWAS transducers in actual SHM:

(1) Predictable and controlled durability of the PWAS transducers on structural materials under environmental attacks and mechanical fatigue loading

(2) Understanding and predictive simulation of PWAS electroacoustic interaction with the structural substrate to achieve active damage detection

(3) Design and analysis of the layered PWAS architectures to achieve low-power ultra low voltage performance for wireless interrogation capability

This paper has presented recent results obtained in the Laboratory for Active Materials and Smart Structures (LAMSS, http://www.me.sc.edu/research/lamss/) in the modeling and predictive simulation of PWAS behavior during structural health monitoring with multi-modal guided waves. Attention has been paid in this paper to three aspects: (a) the convergence of the finite element method (FEM) modeling of guided-wave propagation in thin-wall structures in comparison with analysis and experiments and the diffraction from structural damage. (b) The WaveFormRevealer (WFR) analytical predictive tool for the simulation of multimodal guided wave propagation and interaction with damage. (c) Two-way power and energy transduction analysis in a 2-D geometry using analytical modeling of circular crested guided waves.

It was found that FEM analysis is very useful in visualizing the wave propagation and its interaction with the structural flaw/damage and specimen boundaries but it could be computationally intensive and hence inappropriate for extensive studies for optimum SHM system design. In contrast, the analytical WFR code offers very efficient simulation of various wave propagation scenarios with and without damage.

The transmission and reception of guided waves using PWAS transducers in 2-D geometries is subject to tuning between frequency, wavelengths and PWAS size, such that maxima and minima are observed in carpet plots. In particular, it was found that increasing the PWAS size leads to increased power consumption but does not necessarily lead to an increase in the wave power injected into the structure.

Future work on this subject should focus on the validation and verification $(\mathrm{V} \& \mathrm{~V})$ aspects of analytical and MP-FEM simulation through experiments and cross-code usage.

\section{ACKNOWLEDGMENTS}

The financial support of NSF grant CMMI 0925466, Dr. George Hazelrigg Program Director; N00014-11-1-0271, Dr. Ignacio Perez, Technical Representative; Air Force Office of
Scientific Research \#FA9550-11-1-0133, Dr. David Stargel, Program Manager; are thankfully acknowledged.

\section{REFERENCES}

[1] Giurgiutiu, V. (2008) Structural Health Monitoring with Piezoelectric Wafer Active Sensors, Elsevier Academic Press, 760 pages, ISBN 978-0120887606, 2008

[2] Gresil, M.; Shen, Y.; Giurgiutiu, V. (2011) "Predictive Modeling of Ultrasonic SHM with PWAS Transducers", 8th International Workshop on Structural Health Monitoring IWSHM 2011, 13-15 Sept. 2011, Stanford University, CA

[3] Gresil, M.; Giurgiutiu, V.; Shen, Y.; Poddar, B (2012) "Guidelines for Using the Finite Element Method for Modeling Guided Lamb Wave Propagation in SHM Processes", 6th European Workshop on Structural Health Monitoring, 3-6 July 2012, Dresden, Germany, paper \# Tu.2.E.1

[4] Shen, Y.; Giurgiutiu, V. (2013) "WaveFormRevealer - An Analytical Predictive Tool for the Simulation of Multimodal Guided Waves Propagation and Interaction with Damage", Structural Health Monitoring - an International Journal (under review)

[5] Giurgiutiu, V; Bottai-Santoni, G (2009) "An extension of the shear lag solution for structurally attached ultrasonic active sensors", AIAA Journal, 47(8), pp.1980-1983, 2009

[6] Bin, L.; Giurgiutiu, V. (2010) "Modeling Power and Energy Transduction of Embedded Piezoelectric Wafer Active Sensors for Structural Health Monitoring", SPIE International Symposium on Smart Structures and Materials + Nondestructive Evaluation and Health Monitoring, San Diego, CA, 7-11 Mar. 2010, paper \#764797

[7] Giurgiutiu, V. (2010) "Piezoelectric Wafer Active Sensors for Structural Health Monitoring - State of the Art and Future Directions", PVP2010 ASME Pressure Vessels \& Piping Division Conference, July 18-22, 2010, Bellevue, WA, paper \#PVP2010-25292

[8] Lin, B.; Giurgiutiu, V. (2011) "Simplified 2-D Modeling of Power and Energy Transduction of Piezoelectric Wafer Active Sensors for Structural Health Monitoring", SPIE Vol. 7981, paper \#96

[9] Gresil, M.; Giurgiutiu, V. (2011) "Fatigue Crack Detection in Thick Steel Structures with Piezoelectric Wafer Active Sensors", SPIE Vol. 7983, paper \# 101

Table 1 Simulation Parameters for power and energy analytical modeling.

\begin{tabular}{|c|c|c|}
\hline & $\begin{array}{c}\text { Plate structure } \\
(2024 \mathrm{Al} \text { alloy) }\end{array}$ & $\begin{array}{c}\text { Transmitter } \\
\text { PWAS (PZT-850) }\end{array}$ \\
\hline Radius & $\infty$ & $2-15 \mathrm{~mm}$ \\
\hline Height & $1 \mathrm{~mm}$ & $0.2 \mathrm{~mm}$ \\
\hline Frequency & Frequency sweep $1-1000 \mathrm{kHz}$ \\
\hline Constant Voltage Input & \multicolumn{2}{|c|}{$10 \mathrm{~V}$} \\
\hline
\end{tabular}


Propagating Lamb waves
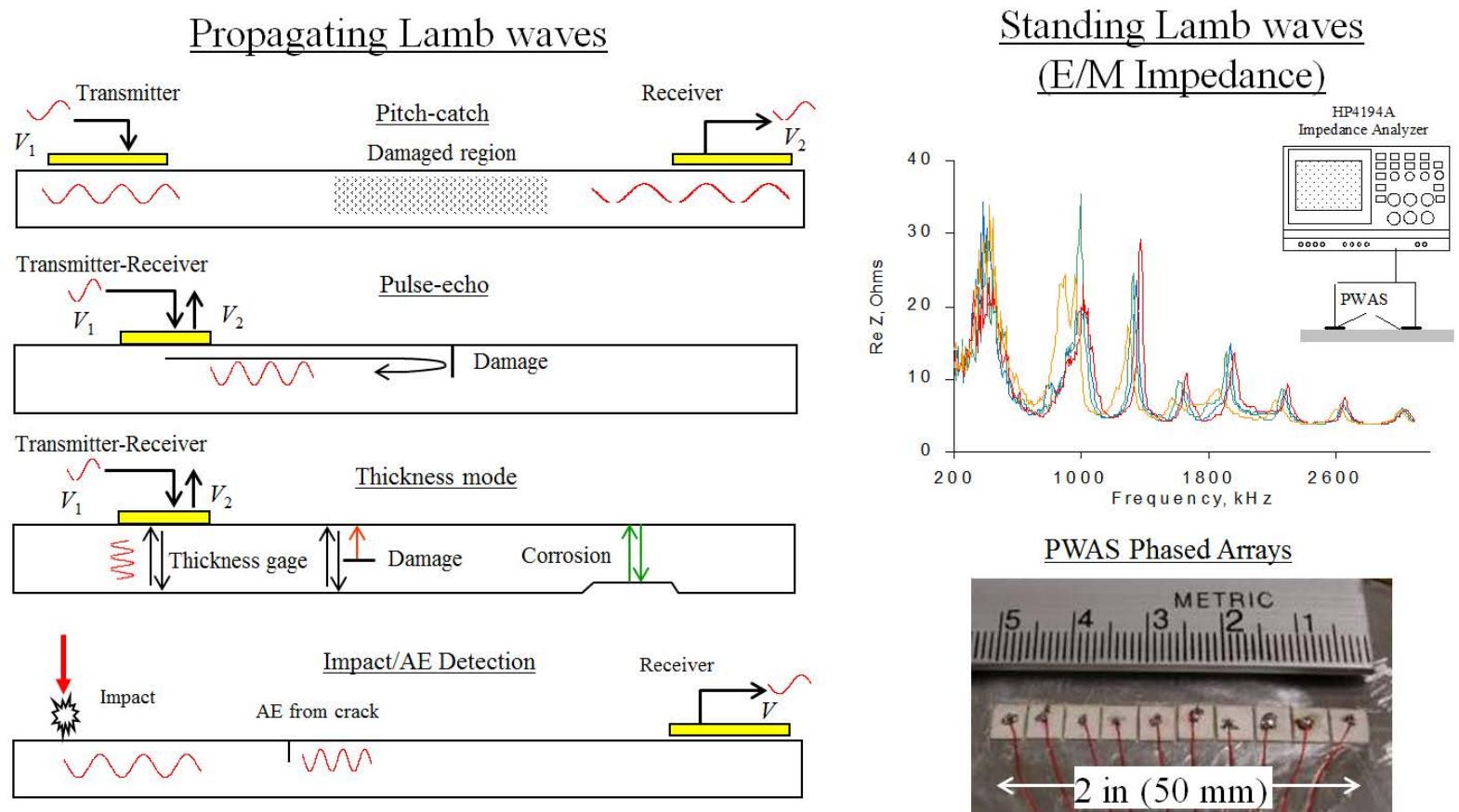

PWAS Phased Arrays

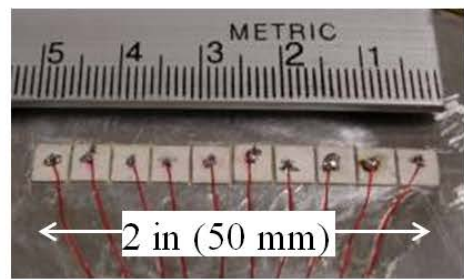

Figure 1 Use of piezoelectric wafer active sensors (PWAS) as traveling wave and standing wave transducers for damage detection in thin-wall structures [1]
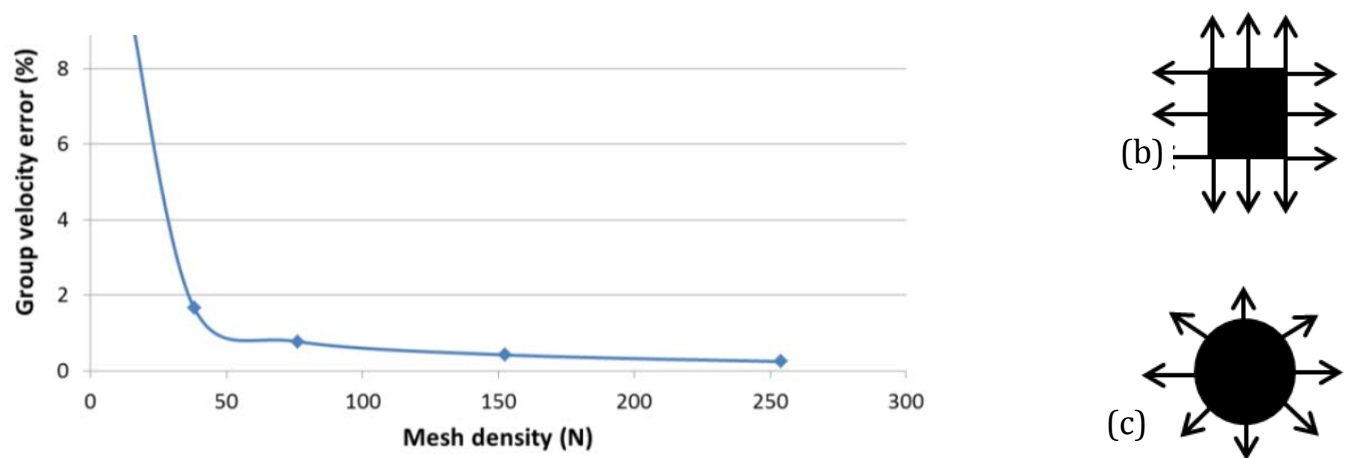

a)

Figure 2: (a) group velocity error versus the mesh density for the A0 mode [2]; self-equilibrating force excitation: (b) for the square PWAS; (c) for the round PWAS [3]
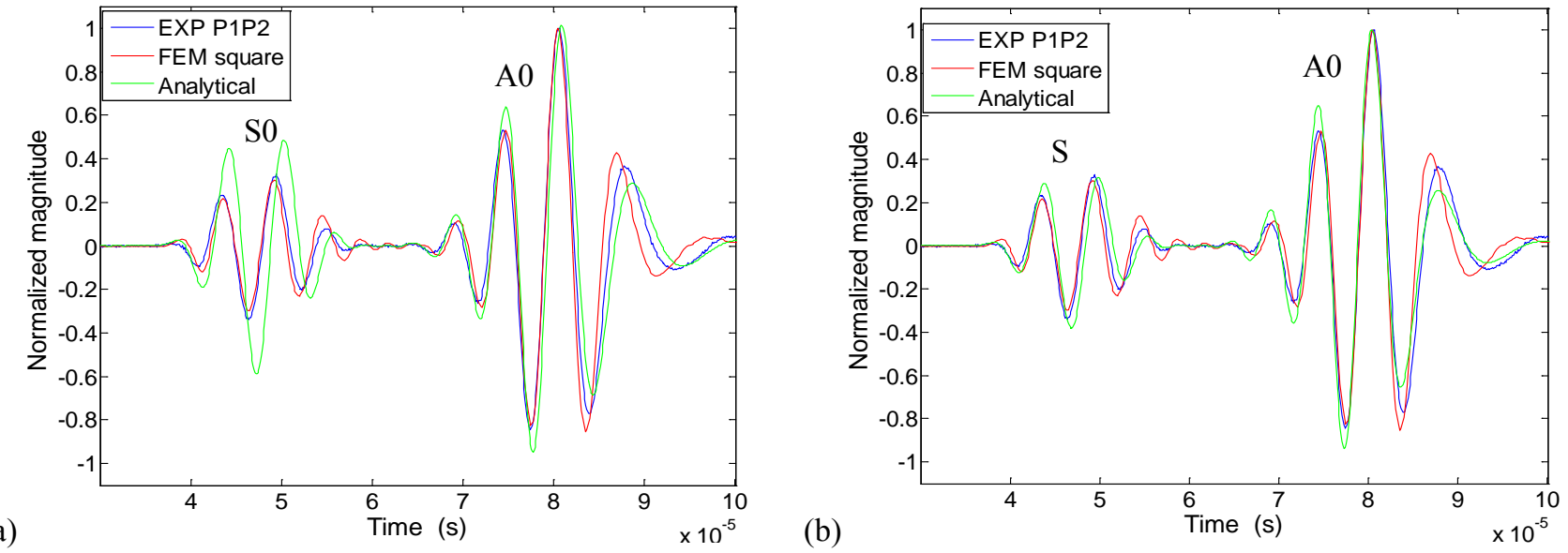
Figure 3: Comparison of the signal receive after a travel of $200 \mathrm{~mm}$ between the analytical, the FEM and the experimental results for the experimental path P1 to P2: (a) under ideal bonding assumption; (b) with shear lag correction [3]

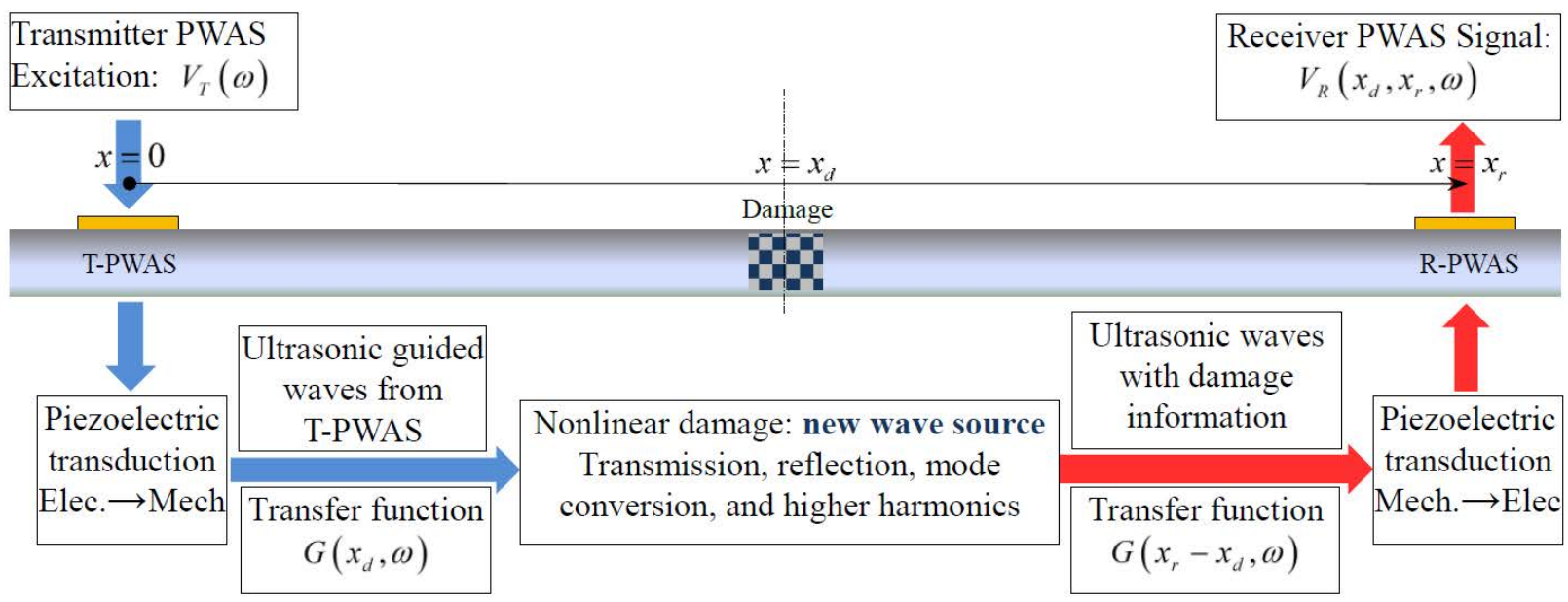

Figure 4: Pitch-catch between a transmitter PWAS and a receiver PWAS with linear damage interaction at $x_{d}$ [4]
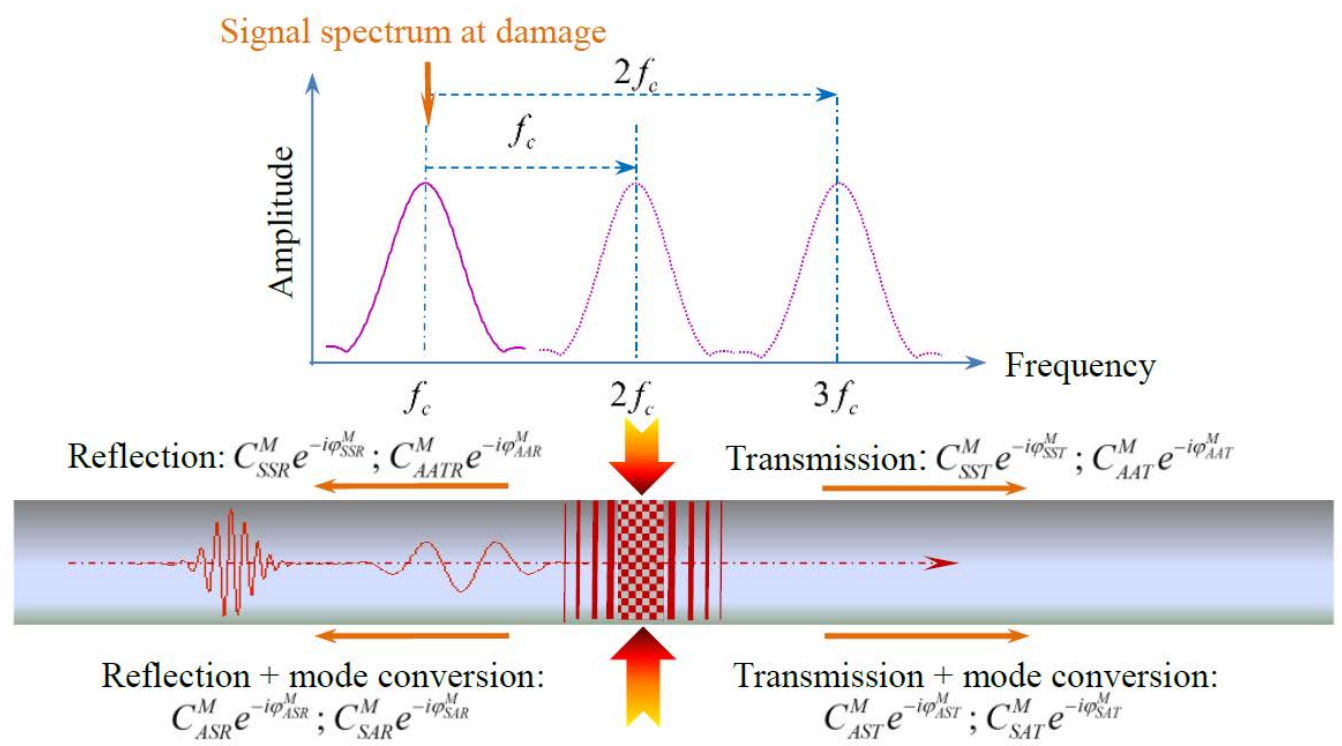

Figure 5: Pitch-catch between a transmitter PWAS and a receiver PWAS with nonlinear damage interaction at $x_{d}$ [4] 


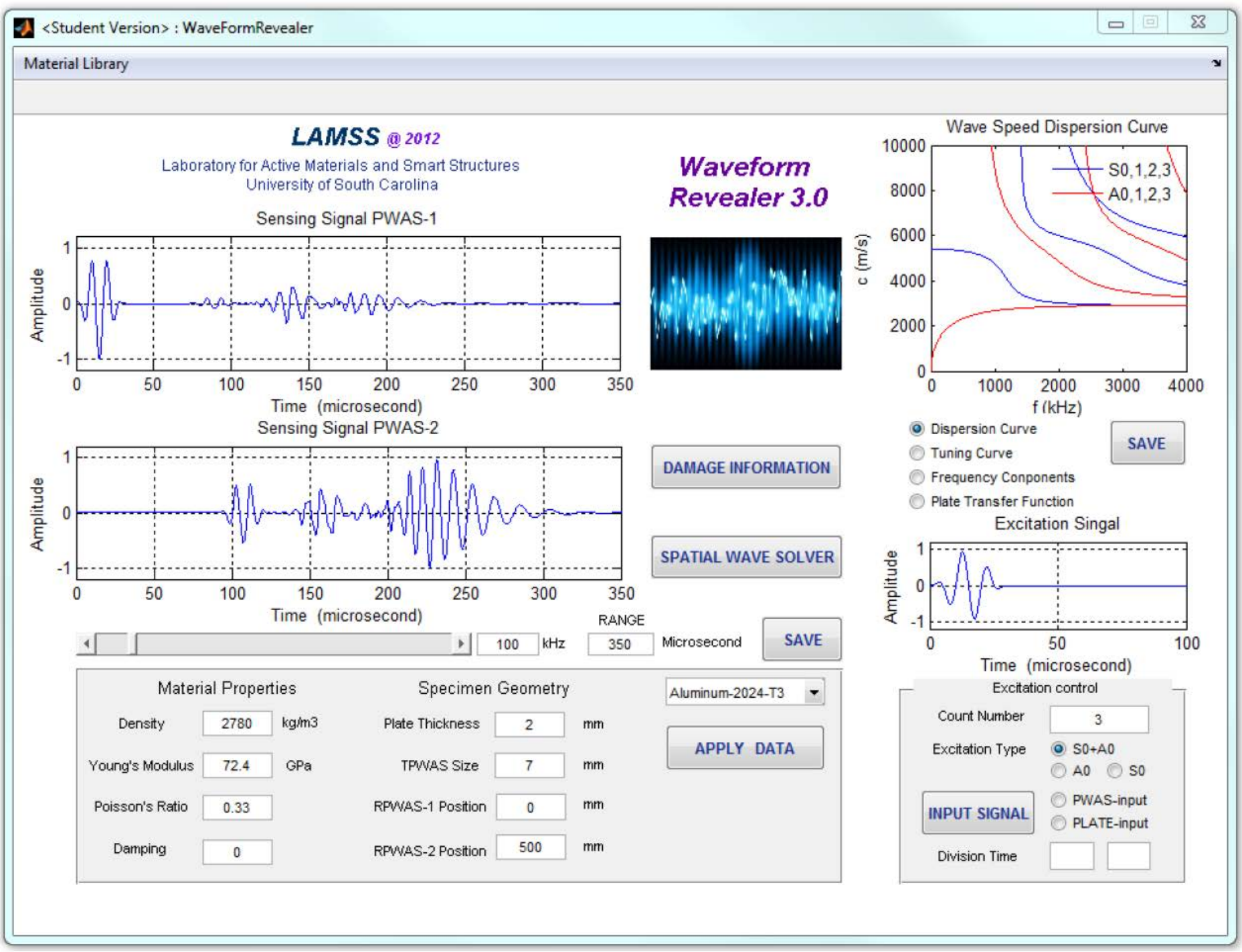

Figure 6: Main GUI of the WaveFormRevealer [4] 


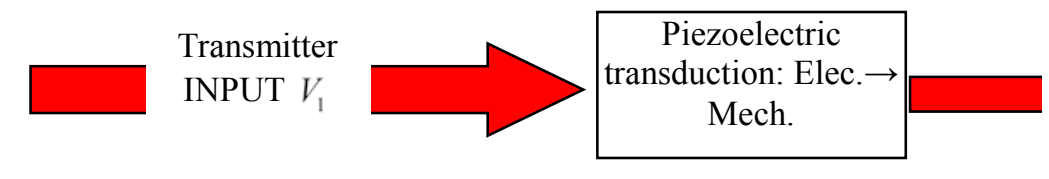

Transmitter PWAS

(Wave Exciter)
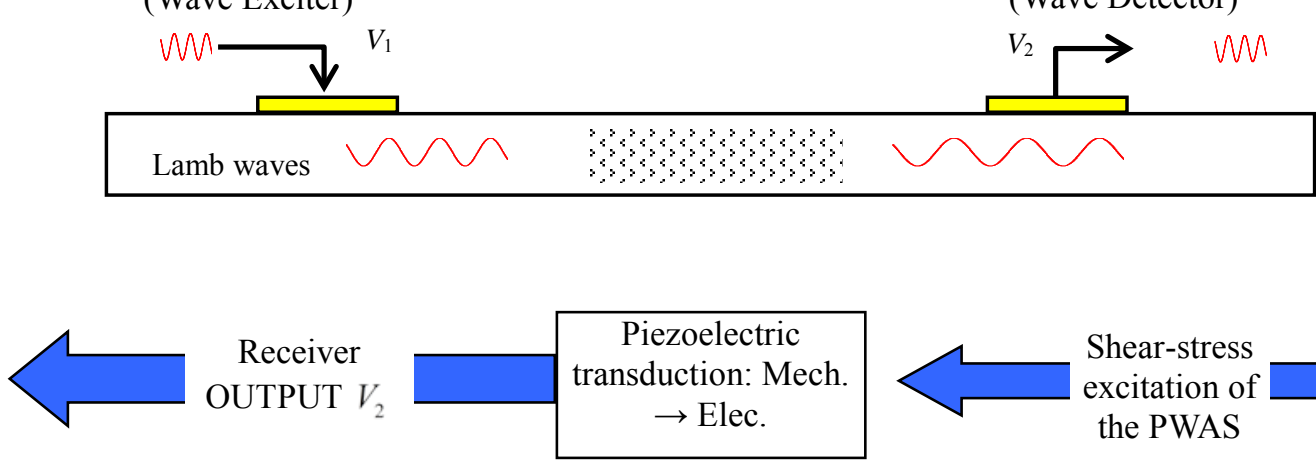

Shear-stress

excitation of the structure

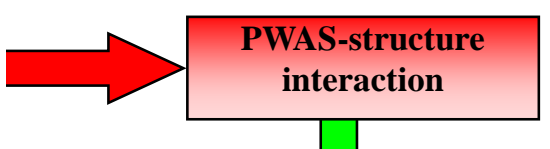

Ultrasonic guided waves from transmitter PWAS

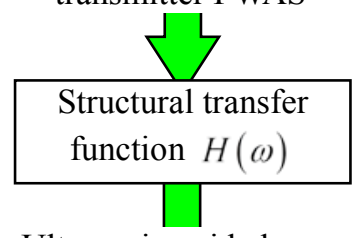

Ultrasonic guided waves arrive at receiver PWAS

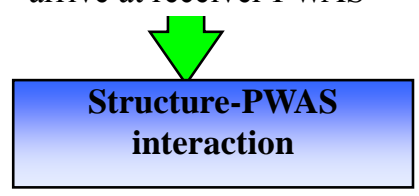

Figure 7 Pitch-catch power flow depicting the various energy transduction stages [6]

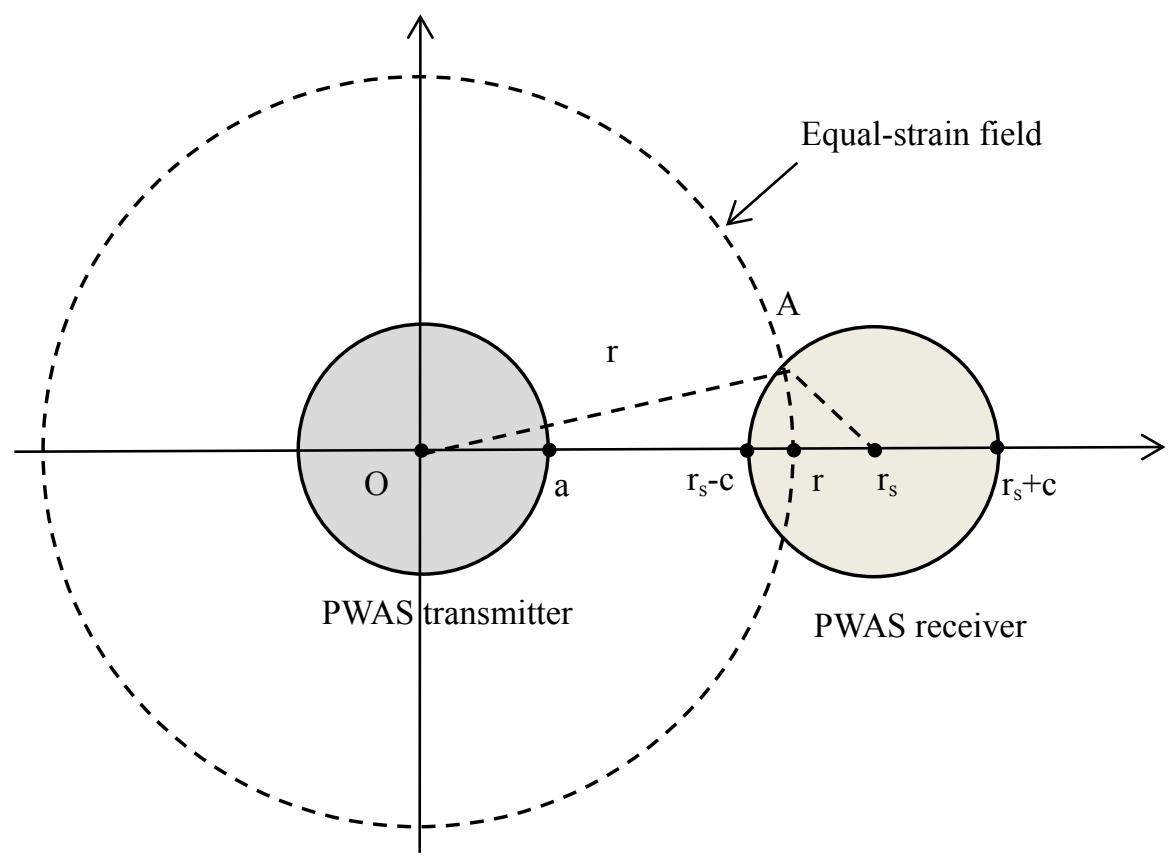

Figure 8 A circular PWAS transmitter with the center at the origin and radius a generates the axial and flexural waves under voltage excitation. The dash circular line represents the equal strain field generated by the PWAS transmitter. A circular PWAS receiver with the center at $\left(r_{s}, 0\right)$ and radius $c$ converts the waves back to voltage. 


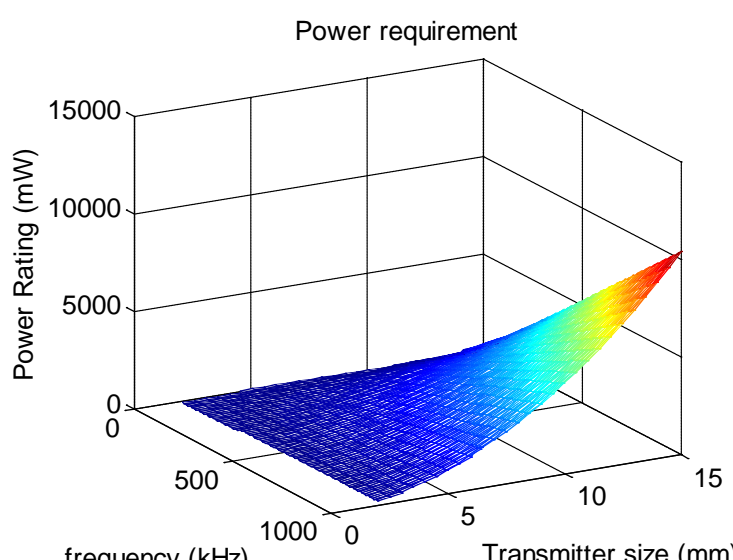

(a)

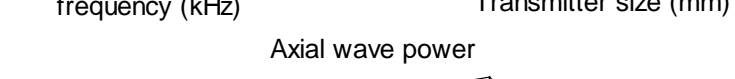

(b)
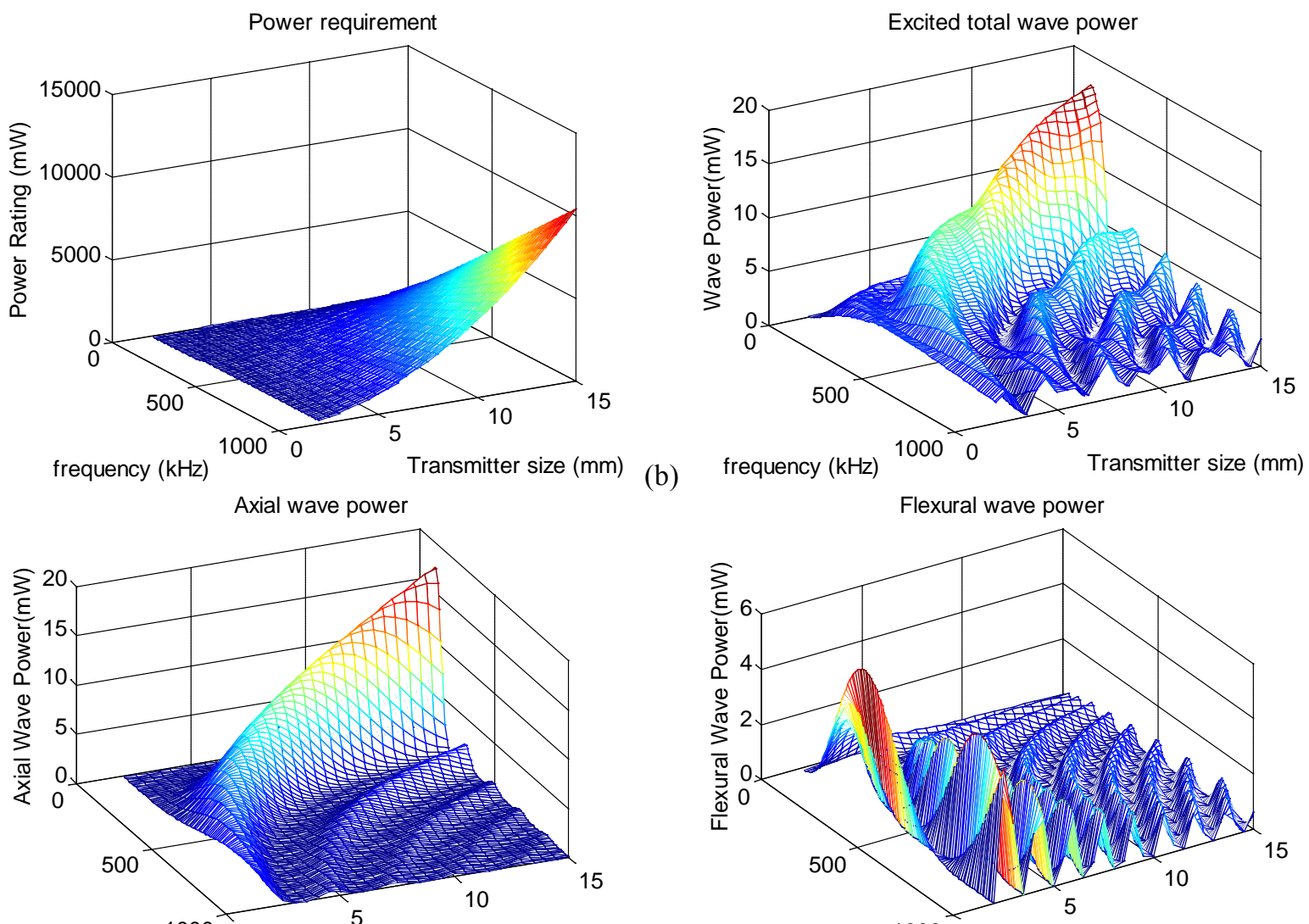

(c) frequency (kHz) $1000{ }_{0} \quad$ Transmitter size (mm)

(d)

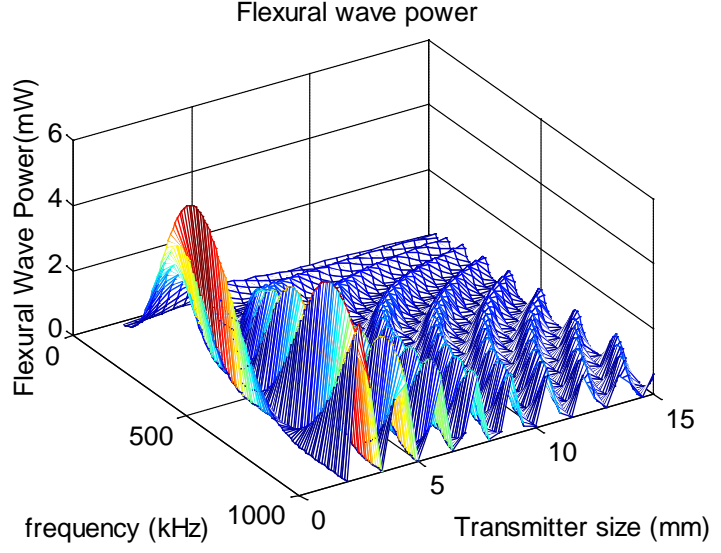

Figure 9 PWAS transmitter under constant voltage excitation (a) power rating; (b) wave power; (c) axial wave power; (d) flexural wave power 